\title{
Porosity of CHA Zeolite Driving the Formation of Polyaromatic Coke Species in the Methanol to Olefins Reaction
}

\author{
Bianca R. Florindo, ${ }^{\oplus a}$ Gabriel L. Catuzo ${ }^{a}$ and Leandro Martins ${ }^{\oplus *, a}$ \\ ${ }^{a}$ Instituto de Química, Universidade Estadual Paulista (Unesp), \\ Prof. Francisco Degni, 55, 14800-900 Araraquara-SP, Brazil
}

\begin{abstract}
The pore size of the chabazite structure is exceptionally suitable for the methanol to olefins (MTO) reaction. The reaction has an induction period required for the formation of a hydrocarbon pool (HP), usually composed of aromatic compounds of different sizes. HP is alkylated by methanol and afterward cracked, leading to the formation of olefins. Despite the importance of HP, its formation and growth in size are exceedingly dependent on the porosity of the catalyst. The ideal is that the formed HP remains stable throughout the reaction because the growth in its size causes blockage of the small catalyst pores, decreasing its capacity. Herein we studied chabazite zeolites with different porosity and structure, caused by variation in the particle size and pores' volume. Porosity influenced the formation of HP species in quantity (as revealed by thermogravimetric analysis (TGA)) and polymerization degree (gas chromatography coupled with mass spectrometer (GC-MS) analysis) and, consequently, the MTO reaction's performance.
\end{abstract}

Keywords: MTO reaction, CHA zeolite, porosity of catalyst, identification of coke

\section{Introduction}

Since the discovery of methanol to hydrocarbons (MTH) and methanol to olefins (MTO) reactions, the interest to produce chemicals based on non-petrochemical routes increases continuously. ${ }^{1-3}$ Solid acid zeolites such as mordenite (MOR), ZSM-5 (MFI), and chabazite (CHA) structures are studied in these reactions. ${ }^{4}$ It stands the CHA structure that has double 6 membered-rings (D6R) and 8 membered-rings (8MR), forming a three-dimensional pore system of large cavities of $0.94 \mathrm{~nm}$ connected by small pore openings with a free opening of $0.38 \mathrm{~nm} .{ }^{5}$ Typically, zeolites of CHA structure are the SSZ-13 (tetrahedrons of Si and $\mathrm{Al}$ only) and $\mathrm{SAPO}-34$ (tetrahedrons of $\mathrm{Si}, \mathrm{Al}$, and P).

The small pores of CHA allow only small molecules to diffuse inside and outside the pores. Methanol and the products propene and ethene have an average kinetic diameter of $0.4 \mathrm{~nm}$. The acidic properties of such structure and its shape-selectivity property turn it an appropriate catalyst to convert methanol into chemicals. ${ }^{6}$ The MTO mechanism consists of establishing an entrapped "hydrocarbon pool" (HP) in the zeolite cavities, formed by the cyclization of the initial scarce quantities of ethene and propene molecules. ${ }^{7}$ For MTO, there is an induction

*e-mail: leandro.martins@unesp.br period comprised of a few seconds, which is necessary for forming the first HP species. ${ }^{8,9}$

HP comprises polyaromatic rings of different sizes possessing a kinetic diameter more extensive than the pore opening. Olefins arise from side alkylation of the aromatic compounds by methanol and subsequent cracking, leading to a large and selective formation of ethene and propene. ${ }^{10-13}$ Recently, a double cycle mechanism for MTO reaction was proposed, where there are two reaction cycles, which are the aromatic and the olefin-based cycle. Ethene would form predominantly through the aromatic-based cycle. Propene and larger alkenes arise from methylation of alkenes or interconversions, such as oligomerization and cracking reactions in the olefin-based cycle., ${ }^{2,14-16}$ Smallpore chabazite zeolites give a higher selectivity to ethene and propene, while medium-pore zeolites, such as ZSM-5, give selectivity to propene and larger molecules, such as pentene. Therefore, the selectivity is dependent on the pore sizes of the zeolites.

Fast deactivation of the catalysts is one of the problems of using zeolites in MTO. It arises due to the uncontrolled growth of the HP formed preferentially close to the surface of the crystals, causing the pore obstruction and consequently the access to the inner active sites, promoting the loss of catalytic activity. ${ }^{17}$ The formation of HP is necessary for the MTO, but when it grows too much (in 
quantity and size), it causes the loss of catalyst activity, and we now refer to it as coke. MTO reactions commonly occur at temperatures between 300 and $500{ }^{\circ} \mathrm{C}$. This feature facilitates the formation of coke since the higher the temperature, the greater the quantity of generated coke. ${ }^{18,19}$ Different strategies can be adopted to extend the life of the catalyst, such as decreasing the particle size or increasing the diameter and volume of the pores in hierarchical catalysts to form connecting channels that minimize the blocking by coke. Dealumination and desilicalization are simple top-down processes intended to generate mesopores in purely microporous catalysts by removing aluminum and silicon atoms from the crystalline structure. It occurs by keeping the zeolite in contact with acid or alkaline solutions and hydrothermal treatments. Alternatively to these processes, it is common to use smaller zeolite crystals synthesized under distinct conditions. ${ }^{20}$ The size of the crystal is an essential issue in several catalytic reactions. The decrease of the crystal leads to better performance since reducing the crystal at the nanometer scale can significantly delay the catalyst's deactivation. ${ }^{1}$

Herein we studied the activity and deactivation of CHA zeolites with varying particle size and porosity, and we associated the zeolite pore volume to the coke species formed in the MTO reaction. We extracted the soluble coke components trapped in the spent catalysts and analyzed them by gas chromatography coupled with a mass spectrometer (GC-MS), providing information on their composition, type, and size.

\section{Experimental}

\section{Preparation of samples}

The zeolites of CHA structure provided by ACS Material, LLC (Pasadena, USA) were SSZ-13 and SAPO-34. Prior to catalytic experiments, the SSZ-13 and SAPO-34 zeolites were ion-exchanged with an aqueous solution of $0.05 \mathrm{~mol} \mathrm{~L}^{-1} \mathrm{NH}_{4} \mathrm{NO}_{3}$, three times, and then calcined at $500{ }^{\circ} \mathrm{C}$ for $2 \mathrm{~h}$ to obtain the acidic zeolites.

The zeolite SSZ-13 was treated (and named MSSZ-13, $\mathrm{M}$ stands for mesoporous) in a solution of $0.2 \mathrm{~mol} \mathrm{~L}^{-1} \mathrm{NaOH}$ for $1 \mathrm{~h}$ at room temperature. Subsequently, ion exchanges were made with ammonium nitrate and the zeolite was calcined, similarly to SSZ-13 and SAPO-34 samples.

\section{Characterization of the zeolites}

$\mathrm{X}$-ray diffraction pattern of the zeolites was registered on a Bruker D8 Advance ECO diffractometer with $\mathrm{Cu}$ $\mathrm{K} \alpha 1$ radiation to determine the purity of catalysts. The data collection occurred in a $2 \theta$ scanning angle between 3 and $40^{\circ}$, at a $0.02^{\circ}$ step, and a counting time of $3 \mathrm{~s}$. The CHA structure was confirmed through the Rietveld method $^{21}$ and the TOPAS ${ }^{\circledR} 4.2$ software $^{22}$ using the CIF (crystallographic information file) presented at the IZA database. The catalyst morphology and the particle size were determined using the Philips XL-30 field emission gun scanning electron microscope, coating the catalysts with gold before measurements. Subsequently, the size of 250 particles was measured using the software Image ${ }^{23}$ to obtain the particle size-frequency histograms. Nitrogen physisorption was used for determining the textural properties, micropore volume, and external specific area. Experiments occurred at $77.3 \mathrm{~K}$ using a Micromeritics ASAP 2420 apparatus. Previously, samples were set to outgas under vacuum at $250{ }^{\circ} \mathrm{C}$ for $12 \mathrm{~h}$. Both micropore volume and external specific area were obtained by the t-plot method using Harkins-Jura equation. ${ }^{24}$

The acid sites of the zeolites were quantified by temperature-programmed desorption of ammonia $\left(\mathrm{NH}_{3}-\mathrm{TPD}\right)$ experiments. Initially, the samples were kept at $300{ }^{\circ} \mathrm{C}$ for $1 \mathrm{~h}$, under a He flow of $60 \mathrm{~mL} \mathrm{~min}^{-1}$ and then cooled down to $100{ }^{\circ} \mathrm{C}$. Then, a flow of $1 \%$ of ammonia in $\mathrm{He}$ was used for $1 \mathrm{~h}$ to saturate the active sites with ammonia. Subsequently, samples were kept for $2 \mathrm{~h}$ at $100{ }^{\circ} \mathrm{C}$ under a He flow to eliminate physisorbed $\mathrm{NH}_{3}$. The desorption measurements were carried out in the range of $100-500^{\circ} \mathrm{C}$ at a rate of $10^{\circ} \mathrm{C} \mathrm{min}-1$. A Pfeiffer vacuum mass spectrometer connected at the reactor out-stream monitored $\mathrm{NH}_{3}$ desorption.

The spent catalysts were characterized by thermogravimetric analysis (TGA) in a flow rate of $100 \mathrm{~mL} \mathrm{~min}^{-1}$ of synthetic air, using a mass of approximately $6 \mathrm{mg}$ of catalyst, with a heating rate of $10{ }^{\circ} \mathrm{C} \mathrm{min}-1$ in the range of $25-1000{ }^{\circ} \mathrm{C}$, in a term scale SDT Q600 V20.9.

The release of the carbonaceous components occluded in the catalyst's pores occurred by dissolving the zeolite at room temperature with $1 \mathrm{~mL}$ of hydrofluoric acid for $1 \mathrm{~min}$, followed by neutralization with sodium bicarbonate. Afterward, coke was extracted with dichloromethane and then analyzed by an Agilent gas chromatograph coupled with a mass spectrometer, gas chromatograph model 7890 , and mass spectrometer model 5977..$^{14,15,17}$

\section{Catalytic tests}

The MTO reaction was performed over the SAPO-34, SSZ-13, and alkali-treated SSZ-13 (MSSZ-13) catalysts using $200 \mathrm{mg}$ of the samples supported on a fixed bed glass reactor placed inside a hot box at $180^{\circ} \mathrm{C}$ to ensure a vapor phase reaction. The reactor was entirely automated and 
controlled from a computer (PID Eng\&Tech microactivity reference). The tests were carried out at $400{ }^{\circ} \mathrm{C}$ for $2 \mathrm{~h}$, using a liquid methanol flow of $0.010 \mathrm{~mL} \mathrm{~min}^{-1}$ that vaporized while passing through the hot box and before reaching the catalyst. Nitrogen flow of $10 \mathrm{~mL} \mathrm{~min}{ }^{-1}$, used as a carrier gas, ensured that reagents and products eluted homogeneously and efficiently through the catalytic bed. The reaction products flowed from the reactor through a valvule that every $10 \mathrm{~min}$ injected the vaporized samples in a Shimadzu gas chromatograph model CG-2014. The analysis system consisted of a Shimadzu SH-RTx-1 crossbond column, coated with a $5 \mu \mathrm{m}$ film of $100 \%$ dimethyl polysiloxane, $30 \mathrm{~m}$ long, $0.53 \mathrm{~mm}$ internal diameter, analysis temperature of $70{ }^{\circ} \mathrm{C}$, and a flame ionization detector. The retention times were found by injecting pure compounds. Selectivities were calculated by quantification of products obtained by comparison with calibration curves. After $2 \mathrm{~h}$ of reaction, methanol feed was interrupted, and the catalyst remained in the reactor for another 15 min under a flow of nitrogen to guarantee the desorption of physisorbed compounds. The objective was to ensure the analysis of only occluded compounds in the study of coke.

\section{Results and Discussion}

\section{Characterization of $\mathrm{CHA}$ zeolites}

The XRD patterns of samples SAPO-34, SSZ-13, and MSSZ-13 in Figure 1a reveal the characteristic patterns of zeolites with the chabazite framework topology (CHA, Figure S1a, Supplementary Information (SI) section). Due to the removal of framework atoms, following distortion of the crystalline structure, and loss of longrange organization, the intensity of peaks of MSSZ-13 depleted. XRD patterns also detected only crystalline phases without diffuse diffraction patterns typical of an amorphous material. Scanning electron microscopy images in Figure 1b likewise confirmed the uniform cubic particles of chabazite topology. The size-frequency depicted in histograms in Figure 1c shows that SAPO-34 particles have an average size of $2.40 \mu \mathrm{m}$ while SSZ-13 $0.18 \mu \mathrm{m}$ and MSSZ-13 0.08 $\mu \mathrm{m}$. MSSZ-13 had somewhat smaller particle sizes compared to SSZ-13 but still keeping the typical cubic shape. The alkaline treatment created not only a secondary porosity into the crystal but also consumed the facets of the cubes proportionally.

Nitrogen adsorption and desorption isotherms in Figure 2a are of type I according to the International Union of Pure and Applied Chemistry (IUPAC) classification for microporous materials. ${ }^{24}$ The treatment of the SSZ-13 sample to create mesopores caused an abrupt drop in the nitrogen adsorption capacity at low pressures and the micropore volume (Table 1). It is a result of the partial and random removal of tetrahedrons from the chabazite crystalline structure. As a consequence, larger pores were formed, as the adsorption capacity in larger $\mathrm{p} / \mathrm{p}_{0}$ significantly increased. Barrett, Joyner, and Halenda (BJH) method applied to the desorption branch the isotherms confirmed that the pristine SAPO-34 and SSZ-13 are mostly microporous materials and that the alkaline process effectively occurred since MSSZ-13 had a broad peak distribution in the mesopore region (Figure $2 b$ ). The t-plots in Figure 2c provided the external area of samples. SAPO-34 had the less inclined slope in the range of $t$ from 0.4 to $1.0 \mathrm{~nm}$, giving an external area of $2 \mathrm{~m}^{2} \mathrm{~g}^{-1}$ (Table 1$)$.
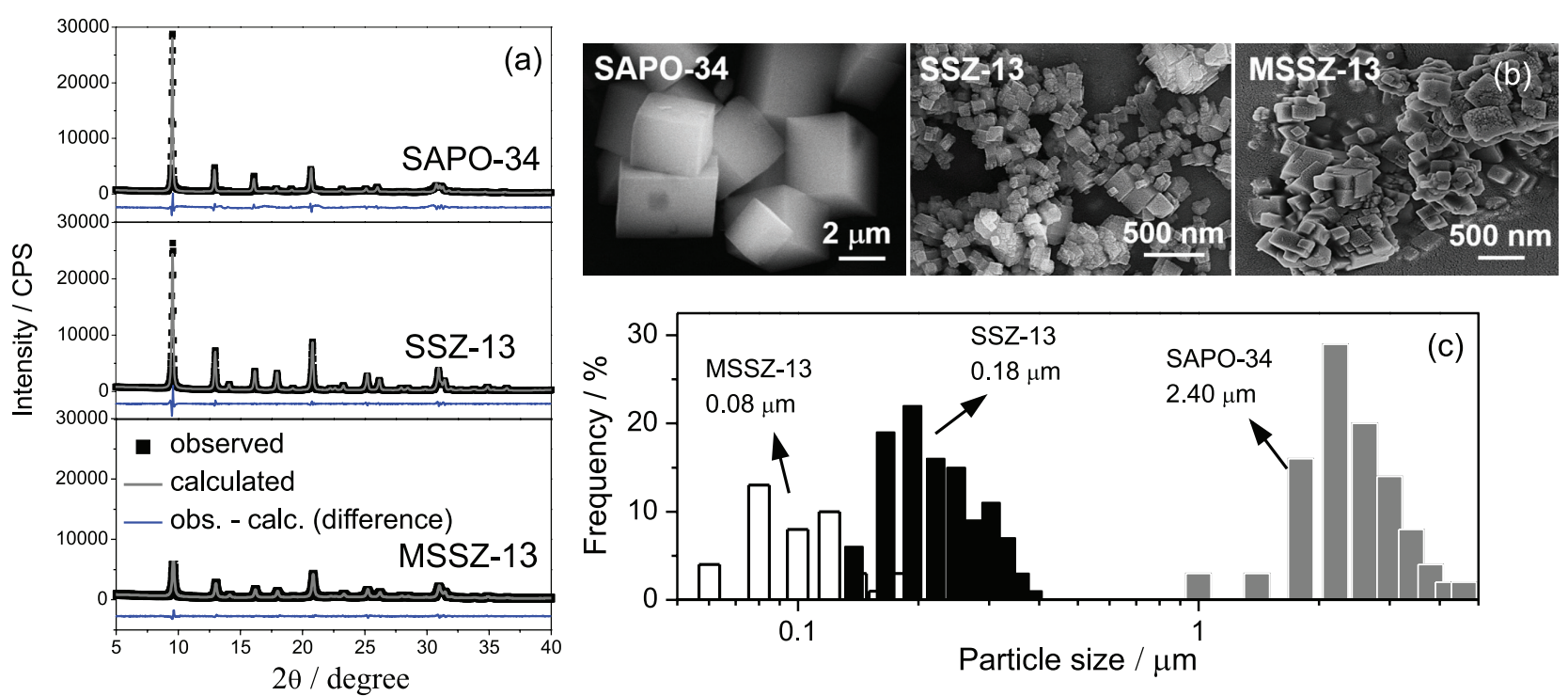

Figure 1. (a) XRD patterns and Rietveld refinement, (b) SEM images, and (c) frequency of sizes of the cubic particles of SAPO-34, SSZ-13, and MSSZ-13. 
Table 1. Textural properties of the CHA zeolites

\begin{tabular}{lcccc}
\hline Sample & $\begin{array}{c}\text { Micropore volume / } \\
\left(\mathrm{mL} \mathrm{g}^{-1}\right)\end{array}$ & $\begin{array}{c}\text { Particle } \\
\text { size } / \mu \mathrm{m}\end{array}$ & $\begin{array}{c}\text { External area t-plot / } \\
\left(\mathrm{m}^{2} \mathrm{~g}^{-1}\right)\end{array}$ & $\begin{array}{c}\text { Estimated external area of } \\
\text { cubes }^{\mathrm{b}} /\left(\mathrm{m}^{2} \mathrm{~g}^{-1}\right)\end{array}$ \\
\hline SAPO-34 & 0.26 & 2.40 & 2 & 1 \\
SSZ-13 & 0.24 & 0.18 & 17 & 15 \\
MSSZ-13 & 0.002 & 0.08 & 50 & 33 \\
\hline
\end{tabular}

${ }^{\mathrm{a}}$ Determined by the mean particle size of histograms of Figure 1c; bdetermined considering the average density of silica of $2.2 \mathrm{~g} \mathrm{~mL}^{-1}$.
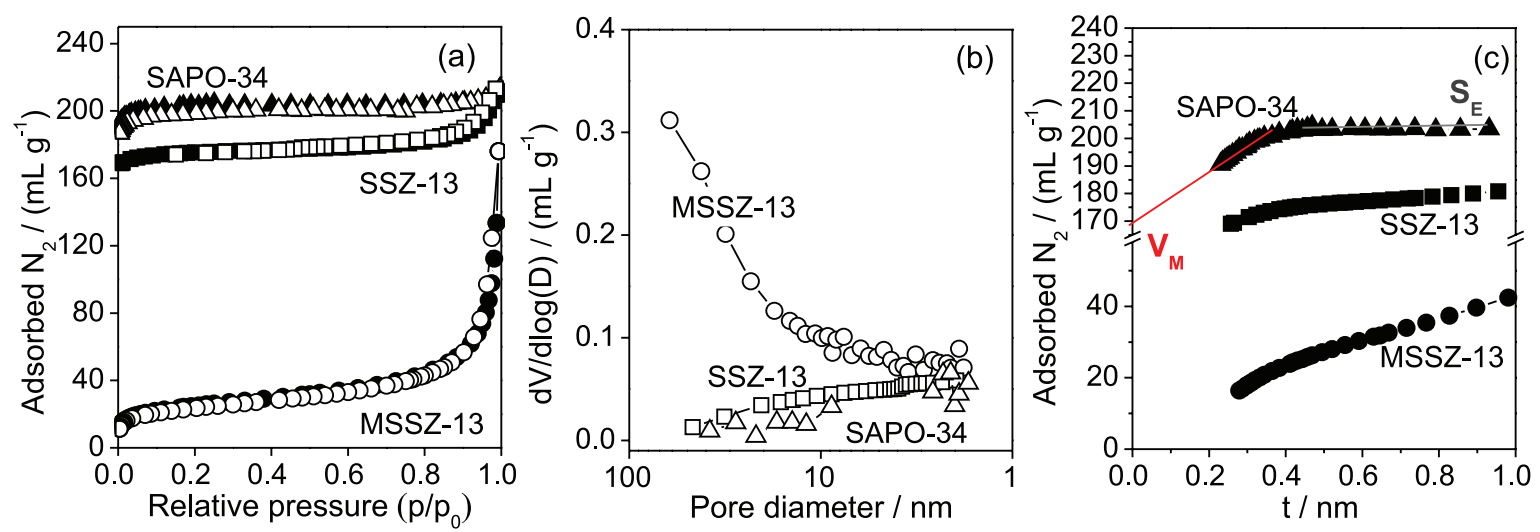

Figure 2. (a) Nitrogen physisorption isotherms, (b) BJH pore size distribution of the desorption branch of isotherms, and (c) t-plot method of SAPO-34, SSZ-13, and MSSZ-13 samples.

The estimation of the surface of the cubic particles, based on their sizes and geometric forms, in comparison to those extracted from nitrogen physisorption experiments, was about the same for SAPO-34 and SSZ-13 samples. MSSZ-13, on the other hand, had an external area of $50 \mathrm{~m}^{2} \mathrm{~g}^{-1}$ in contrast to the estimated external area of $33 \mathrm{~m}^{2} \mathrm{~g}^{-1}$, indicating the creation of pores entered into the crystals. A careful investigation of the micrographs shows that the smooth surface of the SSZ-13 zeolite turned to slightly rough in the MSSZ-13 sample.

The assessment of the acid sites by temperatureprogrammed desorption of ammonia showed that all catalysts had two desorption peaks (Figure 3), one at lower temperatures (LT in Table 2) and another at higher temperatures (HT). The peaks are a consequence of ammonia chemically adsorbed on weak and strong acid sites, respectively. SAPO-34 had the HT desorption peak centered at $450{ }^{\circ} \mathrm{C}$, while SSZ-13 and MSSZ-13 had the HT peak at $500{ }^{\circ} \mathrm{C}$. Brønsted acidity arises in pure aluminosilicate zeolites by introducing charge-balancing protons, making zeolites prevailing solid acid materials.

For $\mathrm{AlPO}_{4}$ frameworks constructed solely from an alternating set of $\mathrm{AlO}_{2}{ }^{-}$and $\mathrm{PO}_{2}{ }^{+}$tetrahedra, the resulting

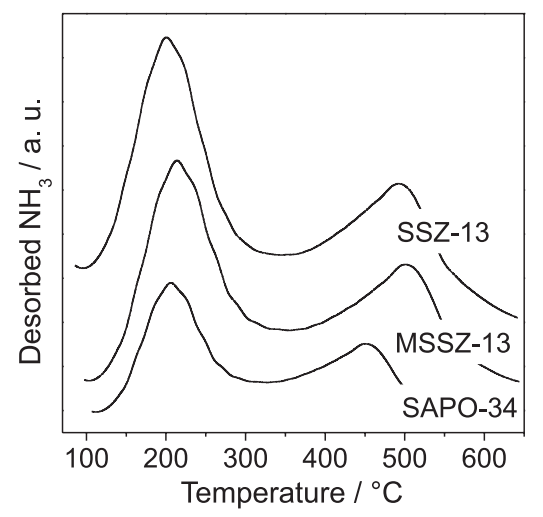

Figure 3. $\mathrm{NH}_{3}$-TPD profiles of the SAPO-34, SSZ-13, and MSSZ-13.

Table 2. Unit cell composition and acidity of CHA zeolites as determined by $\mathrm{NH}_{3}$-TPD

\begin{tabular}{|c|c|c|c|c|c|}
\hline \multirow{2}{*}{ Sample } & \multirow{2}{*}{ Molar composition } & \multirow{2}{*}{$\begin{array}{c}\text { Estimated / } \\
\left(\mathrm{mmol} \mathrm{H} \mathrm{H}^{+} \text {per } \mathrm{g}\right)\end{array}$} & \multicolumn{3}{|c|}{ Acidity by $\mathrm{NH}_{3}$-TPD / $\left(\mathrm{mmol} \mathrm{g}^{-1}\right)$} \\
\hline & & & LT & HT & Total \\
\hline SAPO-34 & $\left(\mathrm{PO}_{2}\right)_{40.5}\left(\mathrm{AlO}_{2}\right)_{49.5}\left(\mathrm{SiO}_{2}\right)_{10.0}$ & 0.88 & 0.31 & 0.19 & 0.50 \\
\hline SSZ-13 & $\left(\mathrm{AlO}_{2}\right)_{7.3}\left(\mathrm{SiO}_{2}\right)_{92.7}$ & 0.62 & 0.48 & 0.16 & 0.64 \\
\hline MSSZ-13 & $\left(\mathrm{AlO}_{2}\right)_{10.1}\left(\mathrm{SiO}_{2}\right)_{89.9}$ & 1.67 & 0.54 & 0.36 & 0.90 \\
\hline
\end{tabular}

$\mathrm{NH}_{3}$-TPD: temperature-programmed desorption of ammonia; LT: low temperature; HT: high temperature. 
framework is neutral. The partial substitution of $\mathrm{P}$ for $\mathrm{Si}$ in the $\mathrm{AlPO}_{4}$ framework, resulting in the SAPO-34, confers negative charges upon the framework, and as occurs in zeolites, Brønsted acidity appears (Figure S1b, SI section). ${ }^{25}$ Silicon incorporation can occur in three mechanisms, named SM1, SM2, and SM3: SM1 occurs when $\mathrm{Si}$ substitutes $\mathrm{Al}$ atoms; SM2 takes place when $\mathrm{Si}$ replaces $\mathrm{P}$ atoms, forming $\mathrm{Si}-\mathrm{O}-\mathrm{Al}$ bond; finally, SM3 occurs when $2 \mathrm{Si}$ atoms substitute both $\mathrm{Al}$ and $\mathrm{P}$ atoms. ${ }^{26}$ SM1 substitution is unfavorable due to the infeasibility of Si-O-P linkages. ${ }^{27}$ For SM2, as phosphorus and silicon charges are $5+$ and $4+$, respectively, their replacement generates a negative charge that, when compensated by a proton, creates a Brønsted site. ${ }^{28}$ For SM3, Si-O-Si bond is formed, which does not create an acid site. However, to avoid the formation of Si-O-P bonds, SM3 substitution occurs concomitantly with a certain degree of SM2 mechanism. It originates aluminosilicate domains (also referred to as Si islands). ${ }^{29}$ Such islands are of particular interest because the acid sites near of such Si aggregates are very strong. ${ }^{28}$ Therefore, while the amount of acid is maximized through the SM2 substitution, the dual SM2 + SM3 mechanism is responsible for forming stronger acid sites. Thus, a proper balance between strength and the number of acid sites can be obtained using an appropriate synthesis method. ${ }^{29}$ Nevertheless, SAPO-34 has weaker acid sites than SSZ-13 zeolite. ${ }^{6,30}$ It reduces hydrogen transfer reactions for SAPO-34 in MTO reaction, reducing the coke formation. ${ }^{31}$ However, the comparison of catalytic activity between SAPO-34 and SSZ-13 should be made when the crystals are approximately the same size, as porosity also significantly affects the results.

For the SAPO-34 zeolite, a low Si/Al molar ratio corresponds to a low acid density, while a high $\mathrm{Si} / \mathrm{Al}$ ratio for the SSZ-13 zeolite is related to a low acid density. Therefore, comparing the $\mathrm{Si} / \mathrm{Al}$ ratios of SAPO-type zeolites and aluminosilicate does not make sense due to the high content of $\mathrm{P}$ atoms in the structure of the first materials.

Acid sites quantification depicted in Table 2 (LT plus HT) shows that SAPO-34 had a reduced amount of acid sites compared to the expected stoichiometric composition obtained by chemical analysis $\left(0.50\right.$ against $\left.0.88 \mathrm{mmol} \mathrm{g}^{-1}\right)$. It could be explained by the diffusion-limited transport of ammonia through the $0.38 \mathrm{~nm}$ pore opening of the CHA structure, but ammonia has a kinetic diameter of $0.34 \mathrm{~nm}$. The explanation, therefore, relies on the topological features, and the adjacent location protons in the SAPO-34 hampering chemisorption of ammonia due to steric hindrance. ${ }^{32}$ For the SSZ-13, the quantification approached the expected value predicted by the molar composition, but not for the MSSZ-13 sample. The explanation for this difference between the expected amount of acidic sites and the measured amount was obtained by ${ }^{27} \mathrm{Al}$ magic angle spinning nuclear magnetic resonance (MAS-NMR) (Figure S2, SI section). During the treatment in $\mathrm{NaOH}$ solution, there is an inevitable removal of $\mathrm{Al}$ from the zeolite structure. However, it is less soluble than silicon species and tends to remain deposited on the catalyst's external surface. According to the NMR spectra, the single peak around $55 \mathrm{ppm}$ is a consequence of tetrahedrally coordinated framework aluminum in zeolites. For MSSZ-13 sample, this peak broadens because of the higher contribution of tetrahedrally distorted Al species. Extra-framework aluminum species that appear in the chemical shift between 30 and -10 ppm increased from 15 to $21 \%$, revealing that acid sites partially converted from the protonic, typical of zeolites, to less acidic extra-framework aluminum oxides.

Catalytic activity and analysis of coke in the spent catalysts

Figure 4 depicts the catalytic results for the MTO carried on the chabazite zeolites. For the SAPO-34 sample, methanol conversion declined sharply in $30 \mathrm{~min}$ of reaction, going from 99 to $40 \%$, remaining steady for

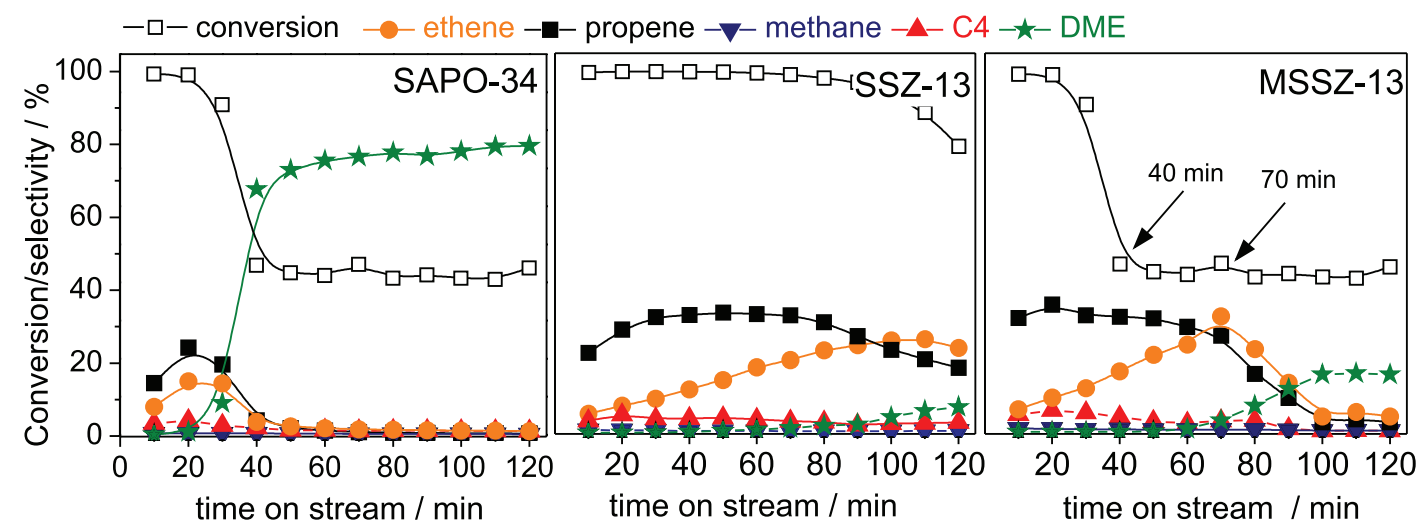

Figure 4. The catalytic performance in the MTO reaction for $2 \mathrm{~h}$. Open symbols refer to methanol conversion and closed symbols to products' selectivity: ethene, propene, methane, C4 (but-1-ene, 2-methylprop-1-ene, etc.) and DME (dimethyl ether). 
the rest of the reaction time. The deactivation is expected in CHA structures; however, a sharp decrease suggests the yolk-shell obstruction of the pores. ${ }^{14,16,17,33-35}$ The formation of carbonaceous compounds in large zeolite crystals covers not only the acid sites, ${ }^{36,37}$ but also blocks pore entrances affecting the diffusion of methanol into inner pores. ${ }^{4,38,39}$ The remaining and stable conversion of methanol occurred at the external acid sites, and dimethyl ether (DME) was the main product. Dehydration of methanol to DME is a common methanol product, usually occurring at low temperatures. In confined zeolite pores and at high temperatures, DME likewise converts into olefins following the HP cycle. As a consequence of the premature deactivation and pore blockage, the formation of ethene and propene was insignificant.

The conversion of methanol on SSZ-13, on the other hand, was constant for $90 \mathrm{~min}$, and then it underwent a slight decrease until the reaction was interrupted, going from 93 to $79 \%$. Several studies ${ }^{36,40,41}$ show that reducing the size of crystals increases the catalyst lifetime. Contrary to what happened to SAPO-34 zeolite, methanol conversion decreased smoothly. The propene's selectivity was initially around $30 \%$, later reducing to $17 \%$ as ethene selectivity increased. The propene to the ethene ratio has an established connection to the free space inside the pores of catalysts. Since HP grows, the free space inside pores decreases, leading to different selectivities. ${ }^{42}$ Both the catalyst and hydrocarbon pool compounds control the olefins production. So, the zeolite properties and size and nature of HP species occluded in the pores determine the selectivity. The change in selectivity is related to the aging of HP that grows due to reaction with methanol or to coupling with neighboring aromatic chains. The occurrence of an induction period for ethene production, but not for propene, is a fact of particular interest. As coke content increases, the selectivity to ethene increases because HP grows to bi- and tricyclic compounds. ${ }^{4,43}$ The kinetic diameter of representative polycyclic aromatic hydrocarbons estimated in Table S1 (SI section) confirms that trimethyl benzene (the smaller aromatic formed in the HP) has a diameter of $0.6 \mathrm{~nm}$, i.e., much larger than the pore entrance of $0.38 \mathrm{~nm}$ and, therefore, do not leave pores during the reaction. Coupled with $\mathrm{NH}_{3}-\mathrm{TPD}$, it is evident that porosity and the constraints imposed by particle size influences the formation of HP species, MTO activity, and selectivity more than acidity.

Porosity plays a decisive role in sample MSSZ-13 that deactivates rapidly, such as occurred with the SAPO-34. The degeneration of micropores (that dropped from 0.24 to $0.002 \mathrm{~mL} \mathrm{~g}^{-1}$ ) after the alkaline treatment explains the deactivation. The scarcely remaining micropores, subjected to the same reaction conditions (methanol flow and temperature), are more prone to early deactivation. However, a careful analysis of selectivity of sample MSSZ-13 reinforces the HP cycle because, despite the reduced conversion of methanol at $40 \mathrm{~min}$, the production of olefins went over up to $70 \mathrm{~min}$. The aromatic species, formed in the early reaction period, grow by continual methylation and then eliminated ethene and propylene, restoring the hydrocarbon pool species and continuing the catalytic cycle. Therefore, due to the cocatalyst action of the HP species, it is named as an autocatalytic reaction. The presence of mesopores, which provided a different degree of confinement and impediment, guaranteed an extended lifetime for the catalyst. DME formation starts just after $80 \mathrm{~min}$ on stream. There must be a point of maximum performance for SSZ-13 and MSSZ-13 samples, that is, the presence of mesopores in the sample, but preserving most of the microporosity. Some studies ${ }^{44}$ on alkaline treatments show that this occurs for samples with a $\mathrm{Si} / \mathrm{Al}$ ratio of around 40 ; however, the chabazite structure typically has ratios below 20 . Therefore, one way to obtain such a sample is through zeolite synthesis with designed structure-directing agents and specific synthesis conditions. ${ }^{45}$

TGA of spent catalysts in Figure 5a shows that they had different quantities of retained coke. The sample SSZ-13 had 19.7 wt.\%, compared to 13.6 wt.\% of SAPO-34, indicating that it does not reflect the deactivation of catalysts. Still, it emphasizes that the location of pores, driven by the porosity of samples, is more critical for the overall performance. Derivative thermogravimetry (DTG) in Figure $5 \mathrm{~b}$ shows that samples conceivably had HP species of diverse sizes because of shifted maximums. Figure 6 shows the GC-MS chromatograms of the $\mathrm{CH}_{2} \mathrm{Cl}_{2}$ extracts, organized together the compounds determined by their mass spectra (not shown) compared with the NIST database. The first marked area indicates monocyclic aromatic compounds (alkyl aromatics) found only in SAPO-34 zeolite. Bicyclic, tricyclic, and polycyclic molecules are represented in the subsequent regions. According to the literature, ${ }^{43} \mathrm{HP}$ species are divided into active and inactivate. Both affect the diffusion of chemicals and deactivation, but some of them might be inactive. For MTO reaction, bicyclic species, such as naphthalenes, have activity in producing light olefins (ethylene and propene). Tricyclics and polycyclics are less active and promote the decrease of the catalyst activity and block the pore entrances. ${ }^{43}$ Figures 6 and S3 (SI section) show that SAPO-34 had a significantly high quantity of less active HP, which reinforces the low selectivity to olefins. HP did not grow further because the catalyst deactivated prematurely cause by large particles and avoided diffusion 

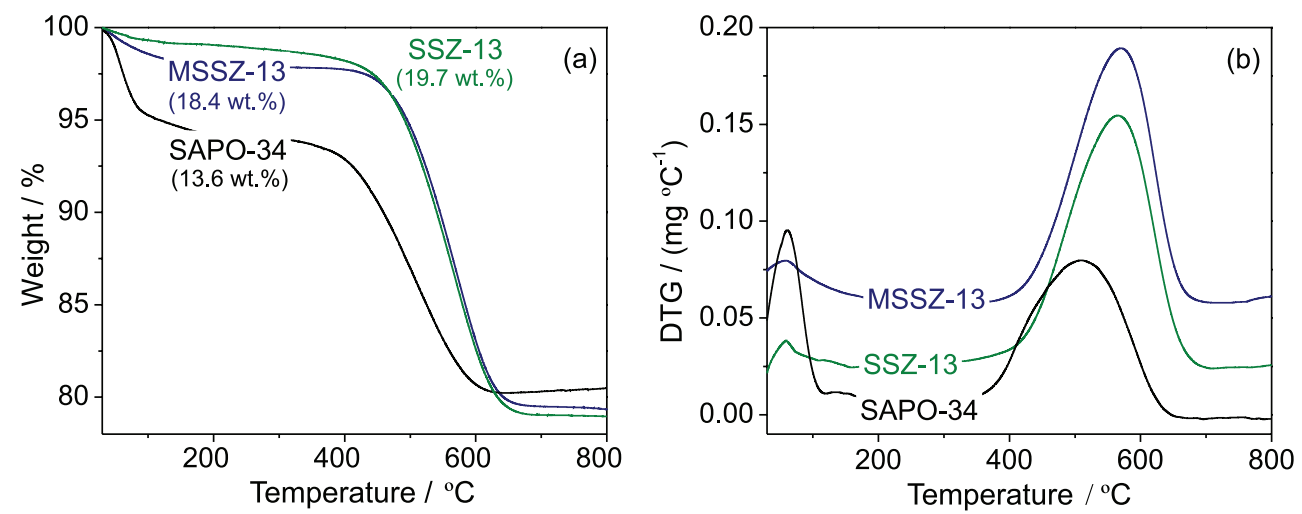

Figure 5. Thermogravimetric analyses, (a) weight and (b) differential thermal gravimetry.
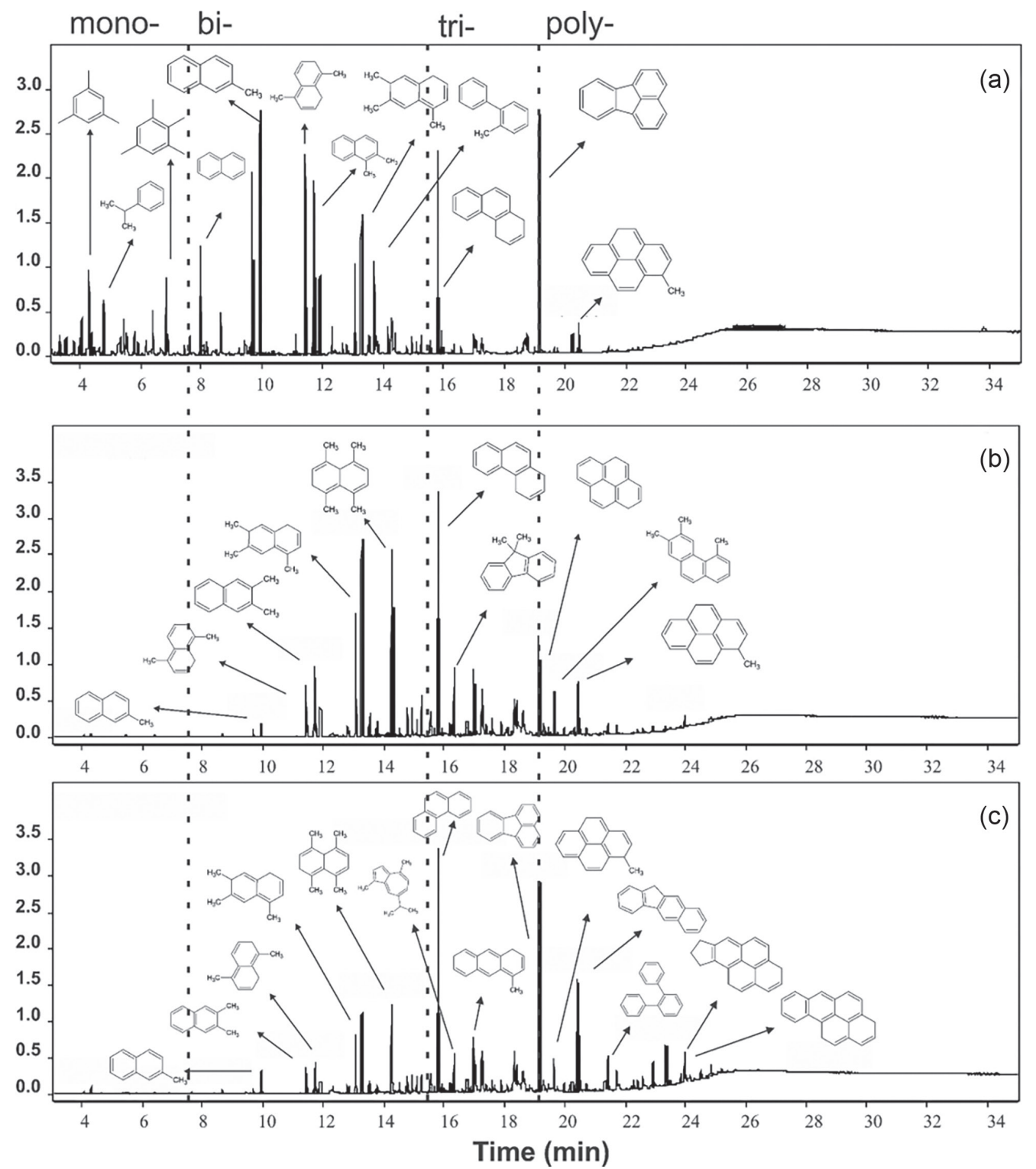

Figure 6. GC-MS chromatograms specifying the mono-, di-, tri- and polycyclic aromatic hydrocarbons retained in the chabazite pores after the MTO reaction for (a) SAPO-34; (b) SSZ-13 and (c) MSSZ-13 zeolites.

of fresh methanol into inner and not accessible pores. SSZ-13, on the other hand, that had mostly bicyclic and tricyclic aromatic compounds, was an active and longerlived catalyst. MSSZ-13, due to the availability of pore 
volume, had most of the polycyclic aromatic compounds, such as fluoranthene and pentacyclic compounds.

Finally, this study shows that the performance of catalysts with chabazite structure depends on the combination of porosity, based on the micro-mesoporous structure of the zeolites. It depends on the zeolite particle size: large particles difficult the access of reagents to active HP species placed within micropores. The existence of mesopores is likewise essential, as it acts as a pocket, allowing HP species to remain active for longer, maintaining a high production of olefins, even when micropores are blocked, and the catalyst is no longer active in the conversion of methanol. The use of micro-mesoporous catalysts may be a strategy to prolong the productive life of the catalysts.

\section{Conclusions}

This study used zeolites of CHA structure with different porosities, which were obtained by varying the size of the cubic particle and by an alkaline treatment to form mesopores. Due to the low $\mathrm{Si} / \mathrm{Al}$ ratio, the treatment was not wholly effective. There was the formation of secondary pores; however, the microporosity decreased dramatically. In the MTO reaction, the catalysts had quite different results concerning activity, selectivity, and stability, despite having acid sites in relatively equivalent amounts. The CHA structure, SAPO-34, with the largest particle size, deactivated quickly and was not selective for olefins. The loss of activity was more significant because of the low external area. The catalyst, SSZ-13, on the other hand, was more active. The catalyst with intracrystalline mesopores, MSSZ-13, deactivated prematurely due to the lack of micropores, which provide the appropriate reaction environment for the formation of HP. However, it continued to produce olefins for $30 \mathrm{~min}$, even with low methanol conversion. The results show that there is a compromise between micropores and mesoropores, which for the CHA structure, must be obtained through bottomup synthesis strategies. The catalyst porosity plays a vital role in forming coke species and, consequently, in their overall performance.

\section{Supplementary Information}

Supplementary information is available free of charge at http://jbcs.sbq.org.br as PDF file. It contains a scheme of the pore system of the chabazite structure and protonic sites in SAPO structures, ${ }^{27} \mathrm{Al}$ NMR-MAS spectra of the SSZ-13 and MSSZ-13 samples, the kinetic diameters of products and representative polycyclic aromatic hydrocarbons retained in the chabazite pores after the MTO reaction and the relative intensity between mono-, bi-, tri- and polyaromatic coke for CHA zeolites.

\section{Acknowledgments}

Authors gratefully acknowledge the financial support provided by the Fundação de Amparo à Pesquisa do Estado de São Paulo (grants No. 2018/01258-5 and 2019/09078-9), Conselho Nacional de Desenvolvimento Cientifico e Tecnológico (CNPq No. 306326/2017-5), and CAPES. We acknowledge LCE-UFSCar for the use of microscopy services and access to the TMP scanning electron microscope.

\section{References}

1. Pinilla-Herrero, I.; Márquez-Álvarez, C.; Sastre, E.; Catal. Sci. Technol. 2017, 7, 3892.

2. Olsbye, U.; Svelle, S.; Bjrgen, M.; Beato, P.; Janssens, T. V. W.; Joensen, F.; Bordiga, S.; Lillerud, K. P.; Angew. Chem., Int. Ed. 2012, 51, 5810 .

3. Chang, C. D.; Lang, W.H.; Smith, R. L.; Chem. Informationsdienst 1979, 10, 249.

4. Chen, D.; Moljord, K.; Holmen, A.; Microporous Mesoporous Mater. 2012, 164, 239.

5. Pham, T. D.; Hudson, M. R.; Brown, C. M.; Lobo, R. F.; ChemSusChem 2014, 7, 3031.

6. Regli, L.; Bordiga, S.; Zecchina, A.; Bjørgen, M.; Lillerud, K. P. In Oxide Based Materials: New Sources, Novel Phases, New Applications, vol. 155; Gamba, A.; Colella, C.; Coluccia, S., eds.; Elsevier: Amsterdam, 2005, p. 471.

7. Vandichel, M.; Lesthaeghe, D.; van der Mynsbrugge, J.; Waroquier, M.; van Speybroeck, V.; J. Catal. 2010, 271, 67.

8. Stöcker, M.; Microporous Mesoporous Mater. 1999, $29,3$.

9. Liu, Y.; Müller, S.; Berger, D.; Jelic, J.; Reuter, K.; Tonigold, M.; Sanchez-Sanchez, M.; Lercher, J. A.; Angew. Chem., Int. Ed. 2016, 128, 5817.

10. Olsbye, U.; Bjørgen, M.; Svelle, S.; Lillerud, K. P.; Kolboe, S.; Catal. Today 2005, 106, 108.

11. Mole, T.; Bett, G.; Seddon, D.; J. Catal. 1983, 84, 435.

12. Dahl, I. M.; Kolboe, S.; Catal. Lett. 1993, 20, 329.

13. Kolboe, S.; Acta Chem. Scand. 1986, 40A, 711.

14. Bjørgen, M.; Olsbye, U.; Kolboe, S.; J. Catal. 2003, 215, 30.

15. Magnoux, P.; Roger, P.; Canaff, C.; Fouche, V.; Gnep, N. S.; Guisnet, M. In Catalyst Deactivation, vol. 34; Delmon, B.; Froment, G. F., eds.; Elsevier: Amsterdam, 1987, p. 317.

16. Pinilla-Herrero, I.; Olsbye, U.; Márquez-Álvarez, C.; Sastre, E.; J. Catal. 2017, 352, 191.

17. Lee, S.; Choi, M.; J. Catal. 2019, 375, 183.

18. Wu, L.; Degirmenci, V.; Magusin, P. C. M. M.; Szyja, B. M.; Hensen, E. J. M.; Chem. Commun. 2012, 48, 9492. 
19. Borodina, E.; Kamaluddin, H. S. H.; Meirer, F.; Mokhtar, M.; Asiri, A. M.; Al-Thabaiti, S. A.; Basahel, S. N.; Ruiz-Martinez, J.; Weckhuysen, B. M.; ACS Catal. 2017, 7, 5268.

20. Ravenelle, R. M.; Schübler, F.; Damico, A.; Danilina, N.; van Bokhoven, J. A.; Lercher, J. A.; Jones, C. W.; Sievers, C.; J. Phys. Chem. C 2010, 114, 19582.

21. Wiles, D. B.; Young, R. A.; J. Appl. Crystallogr. 1981, 14, 149.

22. Diffrac Plus TOPAS, TOPAS 4.2; Bruker AXS GmbH, Germany, 2009.

23. Rasband, W. S.; ImageJ; U. S. National Institutes of Health, Bethesda, Maryland, USA, 1997-2018.

24. Galarneau, A.; Villemot, F.; Rodriguez, J.; Fajula, F.; Coasne, B.; Langmuir 2014, 30, 13266.

25. Sastre, G.; Lewis, D. W.; Richard, C.; Catlow, A.; J. Phys. Chem. B 1997, 101, 5249.

26. Iwase, Y.; Motokura, K.; Koyama, T. R.; Miyaji, A.; Baba, T.; Phys. Chem. Chem. Phys. 2009, 11, 9268.

27. Wang, X.; Guo, F.; Wei, X.; Liu, Z.; Zhang, W.; Guo, S.; Zhao, L.; Korean J. Chem. Eng. 2016, 33, 2034.

28. Sastre, G.; Lewis, D. W.; Catlow, C. R. A.; J. Mol. Catal. A: Chem. 1997, 119, 349.

29. Álvaro-Muñoz, T.; Márquez-Álvarez, C.; Sastre, E.; Catal. Today 2012, 179, 27.

30. Bordiga, S.; Regli, L.; Cocina, D.; Lamberti, C.; Bjørgen, M.; Lillerud, K. P.; J. Phys. Chem. B 2005, 109, 2779.

31. Yuen, L. T.; Zones, S. I.; Harris, T. V.; Gallegos, E. J.; Auroux, A.; Microporous Mesoporous Mater. 1994, 2, 105.

32. Silva, L. L.; Cardoso, D.; Sievers, C.; Martins, L.; J. Phys. Chem. C 2020, 124, 2439.

33. Janssens, T. V. W.; J. Catal. 2009, 264, 130.
34. Müller, S.; Liu, Y.; Vishnuvarthan, M.; Sun, X.; van Veen, A. C.; Haller, G. L.; Sanchez-Sanchez, M.; Lercher, J. A.; J. Catal. 2015, 325, 48.

35. Smith, J. V.; J. Chem. Soc. 1964, 3759.

36. Dai, W.; Wu, G.; Li, L.; Guan, N.; Hunger, M.; ACS Catal. 2013, 3, 588 .

37. Dai, W.; Scheibe, M.; Li, L.; Guan, N.; Hunger, M.; J. Phys. Chem. C 2012, 116, 2469.

38. Hereijgers, B. P. C.; Bleken, F.; Nilsen, M. H.; Svelle, S.; Lillerud, K. P.; Bjørgen, M.; Weckhuysen, B. M.; Olsbye, U.; J. Catal. 2009, 264, 77.

39. Li, Y.; Zhang, C.; Li, C.; Liu, Z.; Ge, W.; Chem. Eng. J. 2017, 320, 458.

40. Nishiyama, N.; Kawaguchi, M.; Hirota, Y.; van Vu, D.; Egashira, Y.; Ueyama, K.; Appl. Catal., A 2009, 362, 193.

41. Xu, Z.; Li, J.; Huang, Y.; Ma, H.; Qian, W.; Zhang, H.; Ying, W.; Catal. Sci. Technol. 2019, 9, 2888.

42. Chen, D.; Moljord, K.; Fuglerud, T.; Holmen, A.; Microporous Mesoporous Mater. 1999, 29, 191.

43. Gao, M.; Li, H.; Yang, M.; Zhou, J.; Yuan, X.; Tian, P.; Ye, M.; Liu, Z.; Chem. Eng. J. 2019, 377, 119668.

44. Groen, J.; Development of Hierarchical Porous Zeolites by Dessicalization: Synthesis, Characterization and Application; VDM Verlag Dr. Müller Aktiengesellschaft \& Co: Saarbrüken, Germany, 2009.

45. Possato, L. G.; Diniz, R. N.; Garetto, T.; Pulcinelli, S. H.; Santilli, C. V.; Martins, L.; J. Catal. 2013, 300, 102.

Submitted: October 6, 2020

Published online: January 14, 2021 